\title{
Methotrexate as a Suspected Trigger of Macrophage Activation Syndrome in Juvenile Idiopathic Arthritis
}

\author{
Roy $S^{1}$, Chakrabartty $S^{2}$
}

\begin{abstract}
A four year old girl with systemic onset juvenile idiopathic arthritis (SOJIA) developed macrophage activation syndrome (MAS) shortly after starting methotrexate. We observed methotrexate is a likely trigger of MAS in SOJIA. Possibly, there are only two case reports suggesting methotrexate triggered MAS. We reviewed the literature for possible mechanisms.

Key words: Macrophage activation syndrome; Methotrexate; Systemic Onset Juvenile Idiopathic Arthritis
\end{abstract}

\section{Introduction}

$\mathrm{M}$ acrophage activation syndrome (MAS) is a devastating complication of systemic onset juvenile idiopathic arthritis (SOJIA) with uncertain aetiopathopathogenesis. Ravelli et $\mathrm{al}^{1}$ had suggested that methotrexate may be a triggering factor for MAS in SOJIA although Eraso et $\mathrm{al}^{2}$ and others refuted their observations. Role of methotrexate in inducing MAS in SOJIA is contemplated in the case report.

\section{The Case}

A four year old girl admitted with fever for one year and evanescent rash with multiple joint pains for ten months. She was diagnosed as a case of SOJIA according to ILAR guideline ${ }^{3}$. Child was taking ibuprofen for one month before admission. Upon admission, we classified her as "systemic arthritis with active arthritis" according to American College of Rheumatology guidelines ${ }^{4}$ and started methotrexate. The next day, she developed pruritus and highgrade, non-remittent fever, with abdominal pain and lethargy. Newonset lymphadenopathy and hepatosplenomegaly were observed and fresh tests revealed bicytopenia and low fibrinogen with elevated CRP, transaminases, INR, triglyceride and ferritin (Table1). She was diagnosed to have developed MAS and died four days later.

\section{Discussion}

Ravelli et al $^{1}$ had found methotrexate as a possible trigger of MAS in SOJIA, whereas Sterba et $\mathrm{al}^{5}$ found methotrexate induced MAS in dermatomyositis. Similar to our scenario, Ravelli et al $^{1}$ also found sharp fever and pruritus after 24 hours of introducing methotrexate along with increase of ferritin from 207 to 10143 and other features of MAS within next 72 hours.
${ }^{1}$ Dr. Soumya Roy, MBBS, Post Graduate Trainee in Paediatrics, Institute of Child Health, Kolkata, India. ${ }^{1} \mathrm{Dr}$. Subroto Chakrabartty, MBBS. DCH. MD. Professor of Paediatrics, Institute of Child Health, Kolkata, India.

\section{Address for correspondence Dr. Soumya Roy E-mail: dr.roy85@gmail.com}

\section{How to cite}

Roy S, Chakrabartty S. Methotrexate as a Suspected Trigger of Macrophage Activation Syndrome in Juvenile Idiopathic Arthritis. J Nepal Paediatr Soc 2017;37(1):95-97.

doi:http://dx.doi.org/10.3126/jnps.v37i1.16894

This work is licensed under a Creative Commons Attribution 3.0 License.

\section{(c) (i)}


Table 1: Main blood reports before and after initiating methotrexate

\begin{tabular}{lcc}
\hline Parameters & On admission & After starting Methotrexate \\
\hline Hemoglobin (g/dl) & 7.2 & 6.2 \\
\hline WBC (per cc) & 14400 & 9100 \\
\hline Platelet (per cc) & 468000 & 140000 \\
\hline CRP $(\mathrm{mg} / \mathrm{dl})$ & 70.4 & 147 \\
\hline ESR (mm) & 74 & 14 \\
\hline SGOT (unit/litre) & 36 & 1095 \\
\hline SGPT(unit/litre) & 16 & 118 \\
\hline INR & - & 2.02 \\
\hline Ferritin (nanogram/dl) & 701 & 40990 \\
\hline
\end{tabular}

Besides the temporal association between methotrexate and MAS, we also reviewed the literature to look for biological plausibility.

MAS occurs when certain trigger factors lead to excessive activation of cytotoxic CD8 $+\mathrm{T}$ cells, with hypersecretion of proinflammatory cytokines. NSAIDS, gold salts, sulfasalazine, penicillamine ${ }^{1}$ canakinumab, etanercept and adalimumab used in SOJIA are known to cause MAS, but role of methotrexate is doubtful ${ }^{2}$. Furhman et al. noted methotrexate-induced-alveolitis, with increased CD4+ and CD8+ T-cells in the bronchoalveolar lavage among a few methotrexate-treated patients ${ }^{6}$. Dobrzanski et al $^{7}$ found single-dose-treatment with methotrexate enhanced T-cell mediated type1 responses. Hatachiet $\mathrm{al}^{8}$ reported development of $\mathrm{CD}+$ T-cell lymphoproliferative disorder with EBV genome in a RA patient, during methorexate therapy. Neurathet al ${ }^{9}$. observed that while methotrexate reduced interleukin and TNF production in synovial fluid, the levels of these cytokines were unaffected in peripheral blood. They also observed that TNF production was reduced from T-lymphocytes, but not from macrophages. Also at antirheumatoid doses, although methotrexate decreases pro-inflammatory cytokines, but cell proliferation is negligibly affected. Infact, at anti-rheumatoid dose, the blood concentration of methotrexate is one-tenth its concentration in synovial fluid ${ }^{9}$. Hence, its actions in the joints and in extra-articular tissues may be different. We

\section{References}

1. Ravelli A, Caria MC, Buratti S, Malattia C, Temporini $F$, Martini A. Methotrexate as a possible trigger of macrophage activation syndrome in systemic juvenile idiopathic arthritis. J Rheumatol 2001;28:865-7.

2. Eraso R, Gedalia A, Espinoza LR. Methotrexate as a possible trigger of macrophage activation syndrome in systemic juvenile idiopathic arthritis. J Rheumatol 2002;29:1104; author reply 1104-5. speculate that in few SOJIA patients, methotrexate may actually increase the cytotoxic T-lymphocytes in extraarticular regions, and initiate MAS

Halevey et al. ${ }^{10}$ reported leucocytoclastic vasculitis in the small vessels of a Rheumatoid Arthritis (RA) patient treated with Methotrexate, which proves the potential ability of Methotrexate to induce autoimmune inflammation in RA.

Methotrexate is an anti-folate drug. It is relevant that another anti-folate drug, sulphamethoxazoletrimethoprim, rarely causes DRESS (Drug rash with eosinophilia and systemic symptoms $)^{11}$, which is an acute immune-mediated reaction causing macrophage and T-lymphocyte activation and cytokine release, reminiscent of drug-induced MAS.

Thus we feel that methotrexate is a possible cause of MAS in SOJIA.

\section{Conclusion}

This case report may open the debate whether methotrexate, a common drug, has this potentially lethal side effect in SOJIA. Paediatricians who are well informed of such possibility will have a higher index of suspicion about methotrexate during their daily practice. This will contribute to salvaging more patients as well as enrichment of the medical literature with more similar case reports.

3. Schneider R, Laxer R. Systemic Juvenile Idiopathic Arthritis. The Rheumatologist. May 9, 2012. Available

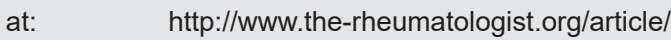
systemic-juvenile-idiopathic-arthritis/ [Date of access: 23/02/2017]

4. Beukelman T, Patkar NM, Saag KG, Tolleson-Rinehart S, Cron RQ, Dewitt EM et al. 2011 American College of Rheumatology Recommendations for the Treatment of Juvenile Idiopathic Arthritis: Initiation and Safety Monitoring of Therapeutic Agents for the Treatment 
of Arthritis and Systemic Features. Arthritis Care Res 201163(4):465-482.

5. Sterba G, Rodriguez C, Sifontes S, Vigilanza P. Macrophage activation syndrome due to methotrexate in a 12-year-old boy with dermatomyositis. J Rheumatol 2004;31:1014-5; author reply 1015.

6. Fuhrman C, Parrot A, Wislez M, Prigent $H$, Boussaud $\mathrm{V}$, Bernaudin JF et al. Spectrum of CD4 to CD8 T-cell ratios in lymphocytic alveolitis associated with methotrexate-induced pneumonitis. Am J RespirCrit Care Med 2001;164:1186-91.

7. Dobrzanski MJ, Reome JB, Hylind JC, Rewers-Felkins KA, Abulsamad K, Adams SL. Ag-specific type 1 CD8 effector cells enhance methotrexate-mediated antitumor responses by modulating differentiated $\mathrm{T}$ cell localization, activation and chemokine production in established breast cancer. Clin Immunol 2008;128:20518
8. Hatachi S, KunitomiA, Aozasa K, Yagita M. CD8(+)T-cell lymphoproliferative disorder associated with EpsteinBarr virus in a patient with rheumatoid arthritis during methotrexate therapy. Mod Rheumatol 2010;20:500-5.

9. Neurath MF, HildnerK, BeckerC, SchlaakJF, Barbulescu K, Germann T. Methotrexate specifically modulates cytokine production by $\mathrm{T}$ cells and macrophages in murine collagen-induced arthritis (CIA): a mechanism for methotrexate-mediated immunosuppression. Clin Exp Immunol 1999;115:42-55.

10. Halevy S, Giryes H, Avinoach I, Livni E, Sukenik S. Leukocytoclastic vasculitis induced by low-dose methotrexate: in vitro evidence for an immunologic mechanism. J EurAcad Dermatol Venereol 1998;10:815.

11. Mona Ben m'rad, Ste'phanie Leclerc-Mercier, Philippe Blanche. et al. Drug-Induced Hypersensitivity Syndrome Clinical and Biologic Disease Patterns in 24 Patients", Medicine 2009;88:131-140. 